\title{
Inverse association between serum antioxidant levels and inflammatory markers is moderated by adiposity: a report based on a large representative population sample of American adults
}

\author{
Mohsen Mazidi ${ }^{1 *}$, Andre Pascal Kengne ${ }^{2}$, Niki Katsiki ${ }^{3}$, Dimitri P. Mikhailidis ${ }^{4}$ and Maciej Banach ${ }^{5,6,7}$ \\ ${ }^{1}$ Division of Food and Nutrition Science, Department of Biology and Biological Engineering, Chalmers University of \\ Technology, SE-41296 Gothenburg, Sweden \\ ${ }^{2}$ Non-Communicable Disease Research Unit, South African Medical Research Council, University of Cape Town, \\ 7505 Cape Town, South Africa \\ ${ }^{3}$ Second Propedeutic Department of Internal Medicine, Medical School, Hippokration Hospital, Aristotle University of \\ Thessaloniki, 54642 Thessaloniki, Greece \\ ${ }^{4}$ Department of Clinical Biochemistry, Royal Free Campus, University College London Medical School, University College \\ London (UCL), London, UK \\ ${ }^{5}$ Department of Hypertension, Chair of Nephrology and Hypertension, Medical University of Lodz, 90-549 Lodz, Poland \\ ${ }^{6}$ Polish Mother's Memorial Hospital Research Institute (PMMHRI), 93-338 Lodz, Poland \\ ${ }^{7}$ Cardiovascular Research Centre, University of Zielona Gora, 65-046 Zielona Gora, Poland
}

(Submitted 16 April 2018 - Final revision received 27 July 2018 - Accepted 8 August 2018)

\section{Abstract}

We examined the association between plasma antioxidant levels and markers of inflammation, including C-reactive protein (CRP) and fibrinogen (FG) in US adults. National Health and Nutrition Examination Survey participants examined between 2001 and 2002 were included, if data on CRP or FG levels. Serum vitamins A and E, two retinyl esters, and six carotenoids were measured using HPLC with photodiode array detection. Multivariable-adjusted linear regression analyses accounted for the survey design and sample weights. A total of 784 eligible participants were included; $47.5 \%$ ( $n$ 372) were men. In multivariable linear regression models, serum $\alpha$-carotene, trans- $\beta$-carotene, cis- $\beta$ carotene, $\beta$-cryptoxanthin, combined lutein/zeaxanthin, trans-lycopene, retinyl palmitate, $\alpha$-tocopherol, retinol and 25 -hydroxy vitamin $\mathrm{D}$ were negatively associated with serum CRP ( $P<0.001$ for all comparisons). Serum $\alpha$-carotene, trans- $\beta$-carotene, cis- $\beta$-carotene, combined lutein/zeaxanthin, trans-lycopene, $\alpha$-tocopherol, retinol and 25-hydroxy vitamin D were negatively associated with serum FG levels ( $P<0.001$ for all comparisons). In the same model, the risk of CVD, defined as CRP levels $>3 \mathrm{mg} / \mathrm{l}$, decreased with increasing levels of antioxidants ( $\alpha$-carotene, trans- $\beta$-carotene, cis- $\beta$-carotene, vitamins A and E). Furthermore, we found a moderate impact of adiposity on the link between antioxidants and CRP. Our results suggest that the lower the antioxidants levels, the higher the inflammatory burden, based on CRP and FG levels. Adiposity moderately affects this association. Furthermore, an inverse relationship between CVD risk and antioxidant levels was observed. This finding suggests that reduced levels of vitamins with antioxidant properties may predispose to increased CVD risk.

Key words: Antioxidants: Inflammation: C-reactive protein: Fibrinogen: Cardiovascular risk: Vitamins

CVD and type 2 diabetes mellitus (T2DM) are typically characterised by oxidative stress, endothelial dysfunction and subclinical chronic inflammation ${ }^{(1)}$. C-reactive protein (CRP) is an acute-phase reactant protein released by hepatocytes following stimulation by inflammatory cytokines, including IL- $6{ }^{(2)}$. Circulating markers of inflammation, such as CRP, TNF- $\alpha$, IL- 6 and IL-1 are associated with a high risk of $\mathrm{CVD}^{(3)}$. It has been suggested that knowledge of CRP levels could improve the prediction of CVD and T2DM occurrence ${ }^{(4)}$. There is also growing evidence that the influence of diet on CVD occurs through mechanisms that include subclinical inflammation ${ }^{(3)}$. CRP might directly promote endothelial dysfunction by decreasing endothelial nitric oxide synthase expression and mRNA stability, stimulating endothelial lectin-like oxidised LDL receptor 1 expression, promoting reactive oxygen species production and enhancing endothelial apoptosis ${ }^{(5)}$.

Abbreviations: 25(OH), 25-hydroxy; CRP, C-reactive protein; FBG, fasting blood glucose; FG, fibrinogen; NHANES, National Health and Nutrition Examination Surveys; SUA, serum uric acid; TC, total cholesterol.

*Corresponding author: M. Mazidi, email mazidi@chalmers.se 
Fibrinogen (FG) is involved in the process of blood coagulation, serving as a major component in thrombosis, and is regarded as one of the inflammatory markers ${ }^{(6)}$. Correlations between FG and CVD risk have been widely investigated. Existing studies support the independent association of elevated FG level with atherosclerotic $\mathrm{CVD}^{(7)}$, as well as recurrent CVD and mortality in patients with existing $\mathrm{CVD}^{(8,9)}$.

A higher intake of fruits and vegetables is associated with a lower risk of $\mathrm{CVD}^{(10)}$, possibly due to the antioxidant properties of several phytochemicals and vitamins that are abundant in fruits and vegetables ${ }^{(11)}$. Antioxidants, including $\alpha$ tocopherol, retinol, $\beta$-carotene, vitamins $\mathrm{D}$ and $\mathrm{E}$ have been reported to affect oxidative stress or inflammatory markers in vitro ${ }^{(12)}$, in rodent models ${ }^{(13)}$ and in epidemiological studies ${ }^{(14)}$. However, interventional studies in humans assessing the effects of a single antioxidant on CVD risk factors have been controversial ${ }^{(15-17)}$.

In the present study, we aimed to evaluate the associations between serum antioxidant levels and inflammatory markers, including FG and CRP, in US adults aged $\geq 18$ years, who took part in the National Health and Nutrition Examination Surveys (NHANES) between 2001 and 2002. Furthermore, we evaluated the impact of adiposity (assessed by BMI) on the link between serum antioxidant levels and inflammatory markers.

\section{Methods}

\section{Population}

The NHANES protocol has been extensively described ${ }^{(18)}$. This is an ongoing programme of cross-sectional surveys conducted periodically by the US National Center for Health Statistics (NCHS). Participants in NHANES (about 5000/year) are selected using a multistage probability sampling approach, with where relevant, oversampling of certain segments of the population. Surveys are approved by the NCHS Research Ethics Review Board and all participants provide informed consent. During these surveys, data on demographics, dietary and behavioural patterns are collected using questionnaires administered during home visits. The interview consists of questions on sociodemographic characteristics (age, sex, education, race and health insurance) and history of diagnosed medical conditions. Anthropometric measurements, physical examination and sample collection for biomarkers assays are performed by trained survey workers using mobile examination units. Height and weight, measured with participants in underwear, are used to calculate BMI as weight in kg divided by the square of height in $\mathrm{m}$. Based on self-reported smoking status, participants are classified as current smokers or not ${ }^{(19)}$. The NHANES data are reported per 2-year cycles and are made publicly available for any relevant purpose.

\section{Biochemical assays}

A blood sample was drawn from an antecubital vein. Serum concentrations of vitamins A (retinol) and E ( $\alpha$-tocopherol), two retinyl esters, and six carotenoids ( $\alpha$-carotene, trans- $\beta$-carotene, cis- $\beta$-carotene, $\beta$-cryptoxanthin, combined lutein/zeaxanthin and trans-lycopene) were measured using HPLC with photodiode array detection ${ }^{(20)}$. Total serum 25-hydroxy $(25(\mathrm{OH})$ ) vitamin $\mathrm{D}$ was assayed using a RIA kit (DiaSorin) ${ }^{(21)}$. The CV was $7 \%{ }^{(21)}$. Glycated $\mathrm{Hb}$ was measured using a Tosoh A1C $2 \cdot 2$ Plus Glycohemoglobin Analyzer (Tosoh Bioscience). Fasting blood glucose (FBG) was measured by using a hexokinase enzymatic method. Insulin was measured using an ELISA immunoassay (Mercodia) ${ }^{(22)}$. Levels of total cholesterol (TC) and TAG were measured enzymatically; LDL-cholesterol was calculated according to the Friedewald equation ${ }^{(23)}$. Serum CRP concentrations were measured by latex-enhanced nephelometry and serum uric acid (SUA) by the uricase-peroxidase technique $^{(19)}$. Based on the NHANES Laboratory Procedures Manual, total bilirubin concentration $(\mathrm{mg} / \mathrm{dl})$ in serum or plasma was measured using a timed-endpoint Diazo method, a colorimetric analysis at $520 \mathrm{~nm}$, and the sensitivity was $0.1 \mathrm{mg} /$ $\mathrm{dl}(1.71 \mu \mathrm{mol} / \mathrm{l})$. Other laboratory-test details are available in the NHANES Laboratory/Medical Technologists Procedures Manual $^{(19)}$. CRP levels $>3 \mathrm{mg} / \mathrm{l}$ was considered as an indicator of high CVD risk ${ }^{(24)}$.

\section{Statistical analysis}

Data were analysed using SPSS complex sample module version 22.0 (IBM Corp.). We followed the Centers for Disease Control and Prevention guidelines for analysis of the complex NHANES data, accounting for the masked variance and using the proposed weighting methodology ${ }^{(25,26)}$. We used mean and standard error of mean for continuous measures and percentages for categorical variables. Adjusted (for age, sex, race, education, marital status, BMI, serum bilirubin, SUA, TAG, TC, FBG and smoking) logistic regressions were used to investigate the associations between antioxidants, CRP and FG, as well as the likelihood of 'CVD risk' with quarters of serum antioxidants (with the first quarter (Q1) considered as reference). Multicollinearity for the multiple linear regressions was assessed with variance inflation factors (VIF) at each step ${ }^{(27)}$. Multi-collinearity was considered high for VIF $>10^{(27)}$. Groups were compared using ANCOVA and $\chi^{2}$ tests.

The SPSS macro for moderation model by Preacher and Hayes $^{(28)}$ was used to investigate the effects of adiposity on the associations of antioxidants with CRP and FG. The application of this macro allowed to simultaneously test the moderation impact of adiposity, while adjusting for relevant extraneous factors. The approach also allowed the visualisation of the impact of each standard deviation change in the potential moderator on the relationship between independent and dependent variables. We tested for the presence of an effect of the adiposity adjusted model (age, sex, race, education, marital status, BMI, serum bilirubin, SUA, TAG, TC, FBG and smoking). All tests were two sided and $P<0.05$ was used to characterise statistically significant results.

\section{Results}

Overall, 784 participants were eligible for this analysis, including $372(47.5 \%)$ men. The mean age was 46.9 years overall, 
with no difference between men and women (47.2 v. 46.6 years, respectively; $P=0.071$ ). Demographic characteristics of the participants across quartiles of CRP and FG are shown in Table 1. Age increased from 40.9 (lowest quartile) to $48 \cdot 1$ (top quartile) years across increasing quartiles of CRP and from 41.5 to $49 \cdot 1$ years across increasing quarters of $\mathrm{FG}(P<0.0001$ for all comparisons). For both CRP and FG, the proportion of women was higher in the top than in the lowest quartiles $(P<0.001$ for all comparisons). Significant differences were observed in the distribution of race, marital status and education, across quartiles of CRP and FG (Table 1).

Age, sex, race, education, marital status, BMI, serum bilirubin, SUA, TAG, TC, FBG and smoking-adjusted mean serum levels of antioxidants across quartiles of CRP and FG are shown in Table 2 . Levels of $\alpha$-carotene, trans- $\beta$-carotene, cis- $\beta$-carotene, $\beta$-cryptoxanthin, combined lutein/zeaxanthin and retinol decreased across increasing quartiles of CRP and FG $(P<0.001$ for all comparisons), whereas concentrations of trans-lycopene and retinyl palmitate were reduced only across quartiles of CRP ( $P<0.001$ for all comparisons). Levels of $\alpha$-tocopherol and $25(\mathrm{OH})$ vitamin D significantly decreased only across increasing quartiles of $\mathrm{FG}$ ( $P<0.001$ for all comparisons).

In multivariable linear regression models adjusted for age, sex, race, education, marital status, BMI, serum bilirubin, SUA, TAG, TC, FBG and smoking, a significant negative association was observed for $\alpha$-carotene, trans- $\beta$-carotene, cis- $\beta$-carotene, $\beta$-cryptoxanthin, combined lutein/zeaxanthin, trans-lycopene, retinyl palmitate, $\alpha$-tocopherol, retinol and $25(\mathrm{OH})$ vitamin $\mathrm{D}$ with CRP $(P<0.001$ for all comparisons, Table 3$)$. Furthermore, $\alpha$-carotene, trans- $\beta$-carotene, cis- $\beta$-carotene, combined lutein/ zeaxanthin, trans-lycopene, $\alpha$-tocopherol, retinol and $25(\mathrm{OH})$ vitamin $\mathrm{D}$ were negatively associated with FG levels $(P<0.001$ for all comparisons). For example, a higher $\alpha$-carotene level by $1 \mu \mathrm{mol} / 1$ correlated with $0.064 \mathrm{mg} / \mathrm{dl}$ lower CRP and $0.043 \mathrm{mg} / \mathrm{dl}$ lower FG levels $(P<0.001$ for all comparisons). Corresponding values were 0.084 and $0.039 \mathrm{mg} / \mathrm{dl}$ for each $\mu \mathrm{mol} / \mathrm{l}$ higher trans- $\beta$-carotene level, 0.073 and $0.049 \mathrm{mg} / \mathrm{dl}$ for each $\mu \mathrm{mol} / \mathrm{l}$ higher cis- $\beta$-carotene, 0.070 and $0.022 \mathrm{mg} / \mathrm{dl}$ for each $\mu \mathrm{mol} / \mathrm{l}$ higher combined lutein/zeaxanthin, and 0.067 and $0.048 \mathrm{mg} / \mathrm{dl}$ for each $\mu \mathrm{mol} / \mathrm{l}$ higher trans-lycopene (Table 3).

Table 4 shows the adjusted logistic regression analysis to determine CVD risk across quartiles of antioxidant vitamins levels. For $\alpha$-carotene, trans- $\beta$-carotene, $\quad$ cis- $\beta$-carotene, vitamins $\mathrm{A}$ and $\mathrm{E}$ levels, the CVD risk decreased with increasing levels of these antioxidants. For example, participants in the top quartiles of vitamins $\mathrm{A}$ and $\mathrm{E}$ had $56 \%$ (95\% CI $0 \cdot 32,0.55)$ and $51 \%(95 \%$ CI $0.41,0.59)$ lower odds of CVD compared with participants in the first quartiles (Table 4).

In adjusted logistic regression analysis, BMI was a significant moderator of the link between CRP and $\alpha$-carotene, trans- $\beta$ carotene, cis- $\beta$-carotene, vitamins A and E. For example, when levels of vitamin A (measured in $\mu \mathrm{mol} / \mathrm{l}$ ) changed from low (1.48) to high (1.98), the CRP in the low BMI category (mean $-1 \mathrm{sD}, 22.4 \mathrm{~kg} / \mathrm{m}^{2}$ ) changed from 0.31 to 0.96 (an increase of 0.65 ). In contrast, in the high BMI category (mean 1sD, $36 \cdot 1 \mathrm{~kg} / \mathrm{m}^{2}$ ), vitamin A (measured in $\mu \mathrm{mol} / \mathrm{l}$ ) changed from 0.34 to 1.12 (an increase of $0 \cdot 78$ ), suggesting that obesity may modulate the impact of vitamin A on CRP.

\section{Discussion}

In the present study, a significant inverse association was observed between serum several antioxidant vitamins and inflammatory markers (i.e. CRP and FG). Furthermore,

Table 1. Demographic characteristics of the participants across quartiles $(\mathrm{Q})$ of $\mathrm{C}$-reactive protein $(\mathrm{CRP})$ and fibrinogen levels (Mean values with their standard errors and percentages)

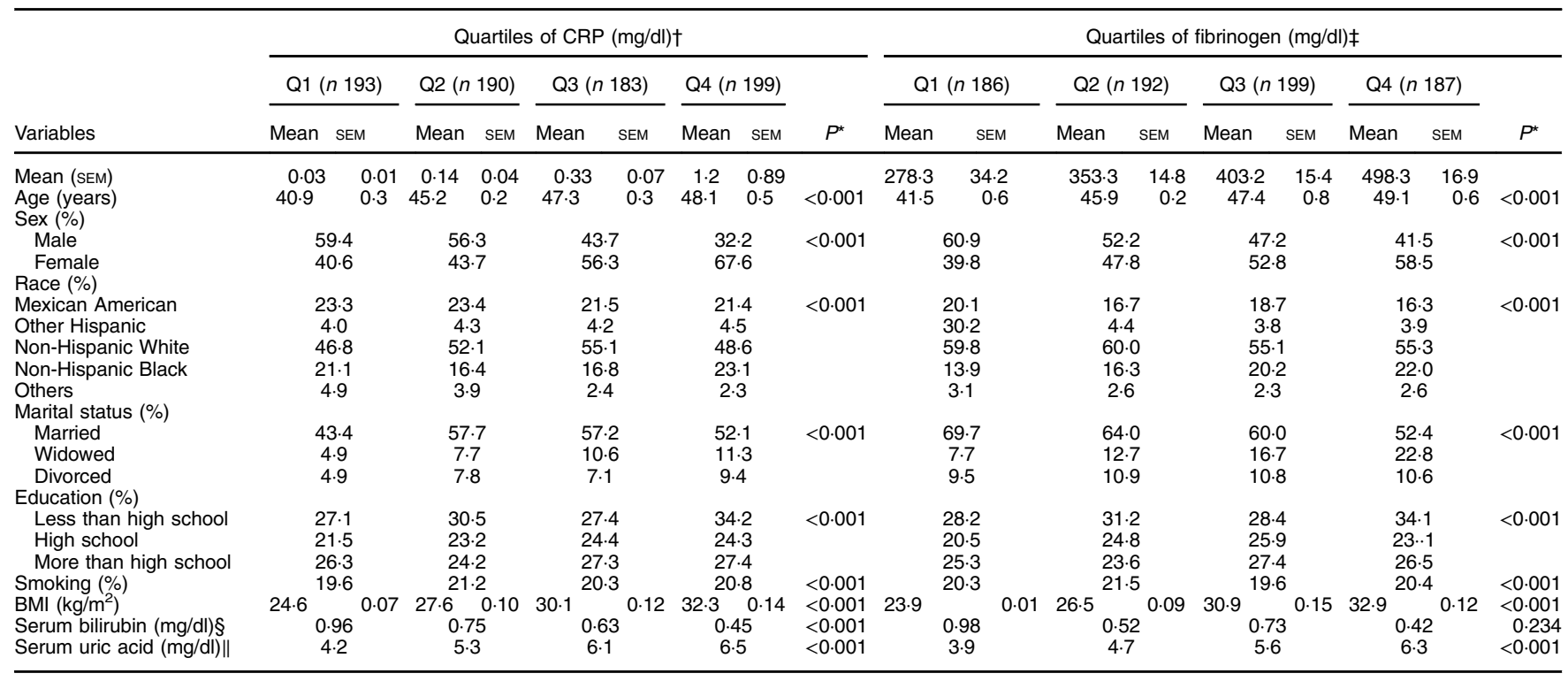

* Variables were compared across quartiles of CRP and fibrinogen using ANOVA or $x^{2}$.

$\dagger$ To convert CRP in $\mathrm{mg} / \mathrm{dl}$ to $\mathrm{mg} / \mathrm{l}$, multiply by 10 .

$\ddagger$ To convert fibrinogen in $\mathrm{mg} / \mathrm{dl}$ to $\mu \mathrm{mol} / \mathrm{l}$, multiply by 0.0294 .

$\S$ To convert bilirubin in $\mathrm{mg} / \mathrm{dl}$ to $\mu \mathrm{mol} / \mathrm{l}$, multiply by 17.1 .

|| To convert uric acid in $\mathrm{mg} / \mathrm{dl}$ to $\mu \mathrm{mol} / \mathrm{l}$, multiply by 59.48 . 
individuals with a higher level of serum antioxidants had a lower risk of CVD (defined by CRP level). These observations were unaffected by the adjustment for several confounding factors, suggesting a potential protective effect of antioxidants against pathological processes involving subclinical inflammation. Furthermore, the link between CRP and antioxidant vitamins levels was mediated by BMI, suggesting a determining role of obesity in the occurrence of subclinical inflammation.

Consumption of selected fruits, vegetables, herbs and spices rich in antioxidants improved markers of oxidative stress and inflammation in a previous review ${ }^{(29)}$. However, there are data not supporting the use of these vitamins to reduce CVD risk ${ }^{(30)}$. In the Women's Health Study ${ }^{(31)}$, no overall benefit was found for vitamin $\mathrm{E}$ in relation to major CVD events and total mortality. The Heart Outcomes Prevention Evaluation trial in people aged $\geq 55$ years with CVD risk factors, showed no overall effect of antioxidant vitamins on CVD outcomes ${ }^{(32)}$. Daily multivitamin consumption did not decrease major CVD events during a decade of follow-up of US men in the Physicians' Health Study II ${ }^{(33)}$. A metaanalysis of randomised trials also reported no effect of antioxidant vitamin supplementation on major fatal and non-fatal CVD, as well as all-cause mortality ${ }^{(34)}$. Furthermore, vitamin E supplementation (at doses $\leq 400 \mathrm{IU} / \mathrm{d}$ ) had no significant impact on inflammatory markers in postmenopausal women ${ }^{(35)}$. In contrast, ex vivo studies reported that vitamin $\mathrm{E}$ at doses of 600-1200 IU/d can significantly decrease the levels of inflammatory factors ${ }^{(36,37)}$.

In all, two studies examined the effects of the combination of vitamins $\mathrm{C}$ and $\mathrm{E}$ on CRP; none found a significant effect on CRP levels ${ }^{(38,39)}$. However, they used different doses, that is, $182 \mathrm{mg}$ $\alpha$-tocopherol and $500 \mathrm{mg}$ vitamin $\mathrm{C}^{(39)}, 371 \mathrm{mg} \alpha$-tocopherol and $515 \mathrm{mg}$ vitamin $\mathrm{C}^{(38)}$. Hartel et al. ${ }^{(40)}$ found that vitamin $\mathrm{C}$ inhibits the lipopolysaccharide-induced IL- 6 and TNF- $\alpha$ production, as well as IL-2 production after phorbol 12-myristate 13-acetate/ionomycin stimulation. It was suggested that vitamin $\mathrm{C}$ could decrease the level of oxidative stress and consequently inflammation, as oxidative damage leads to an inappropriate activation of the transcription $\mathrm{NF}-\kappa \mathrm{B}$ and subsequently to an overexpression of inflammatory proteins ${ }^{(41)}$. Similarly, vitamin $\mathrm{C}$ was shown to inhibit NF- $\kappa$ B activation ${ }^{(42-44)}$.

$\beta$-Carotene is the most investigated carotenoid for its antioxidant activity ${ }^{(45,46)}$. A study which included 14470 current smokers, ex-smokers and never smokers aged $\geq 18$ years who participated in the third NHANES, evaluated the relationship between serum $\beta$-carotene and CRP and reported a strong and inverse association of serum $\beta$-carotene levels with CRP levels ${ }^{(47)}$. Another study that used data from the MacArthur studies of successful aging ( $n$ 672), found a negative link between $\beta$-carotene and CRP concentrations ${ }^{(48)}$. Recently, a study on eighty individuals (mean age $=66.9$ years) reported that the dietary intake of $\beta$-carotene does not significantly affect plasma or salivary CRP levels ${ }^{(49)}$. Another study examined cross-sectional correlations between CRP and plasma levels of $\alpha$-tocopherol and $\beta$-carotene, reporting no association between plasma levels of $\alpha$-tocopherol and $\mathrm{CRP}^{(50)}$. In contrast, plasma $\beta$-carotene was inversely related to $\mathrm{CRP}^{(50)}$.

Vitamin A plays a role in both pro-inflammatory cytokines such as IL-6 and upregulating IL-4 production (which is an anti-inflammatory marker) ${ }^{(51,52)}$. In this context, vitamin A 
Table 3. Adjusted (for age, sex, race, education, marital status, BMI, serum bilirubin, serum uric acid, TAG, total cholesterol, fasting blood glucose and smoking) linear regression for the association between C-reactive protein (CRP) and fibrinogen levels with serum antioxidant vitamins ( $\beta$-Coefficients and $95 \%$ confidence intervals)

\begin{tabular}{|c|c|c|c|c|}
\hline \multirow[b]{2}{*}{ Variables } & \multicolumn{2}{|c|}{ CRP } & \multicolumn{2}{|c|}{ Fibrinogen } \\
\hline & $\beta$ & $95 \% \mathrm{Cl}$ & $\beta$ & $95 \% \mathrm{Cl}$ \\
\hline$a$-Carotene $(\mu \mathrm{mol} / \mathrm{l})$ & -0.06 & $-1.09,-0.39$ & -0.04 & $-0.63,-0.045$ \\
\hline trans- $\beta$-Carotene $(\mu \mathrm{mol} / \mathrm{l})$ & -0.08 & $0.38,-0.19$ & -0.03 & $-0.18,-0.005$ \\
\hline $\operatorname{cis}-\beta$-Carotene $(\mu \mathrm{mol} / \mathrm{l})$ & -0.07 & $-6 \cdot 18,-2 \cdot 78$ & -0.04 & $-3.78,-0.52$ \\
\hline$\beta$-Cryptoxanthin $(\mu \mathrm{mol} / \mathrm{l})$ & -0.07 & $0.73,-0.31$ & -0.02 & $0.35,0.096$ \\
\hline Combined lutein/zeaxanthin $(\mu \mathrm{mol} / \mathrm{l})$ & -0.10 & $-1.02,-0.52$ & -0.07 & $0.68,-0.22$ \\
\hline trans-Lycopene $(\mu \mathrm{mol} / \mathrm{l})$ & -0.06 & $-0.55,-0.23$ & -0.04 & $-0.41,-0.052$ \\
\hline Retinyl palmitate $(\mu \mathrm{mol} / \mathrm{l})$ & -0.02 & $-0.72,-0.03$ & -0.01 & $-0.46,0.23$ \\
\hline Retinyl stearate $(\mu \mathrm{mol} / \mathrm{l})$ & -0.02 & $2 \cdot 23,0.24$ & -0.00 & $-1.03,0.92$ \\
\hline Retinol (vitamin A) $(\mu \mathrm{mol} / \mathrm{l})$ & -0.12 & $0.28,-0.18$ & -0.05 & $-0.14,-0.029$ \\
\hline$a$-Tocopherol (vitamin E) $(\mu \mathrm{mol} / \mathrm{l})$ & -0.11 & $0.27,-0.17$ & -0.05 & $-0.44,-0.056$ \\
\hline 25 -Hydroxy vitamin D (ng/ml) & -0.06 & $0.005,-0.001$ & -0.05 & $0.004,-0.001$ \\
\hline
\end{tabular}

Table 4. Multivariable logistic regression (adjusted for age, sex, race, education, marital status, BMI, serum bilirubin, serum uric acid, TAG, total cholesterol, fasting blood glucose and smoking) for the risk of CVD across quartiles (Q) of antioxidant vitamin levels

(Odds ratios and 95\% confidence intervals)

\begin{tabular}{|c|c|c|c|c|c|c|c|}
\hline \multirow[b]{2}{*}{ Variables } & \multicolumn{3}{|c|}{ CVD risk } & \multirow[b]{2}{*}{ Variables } & \multicolumn{3}{|c|}{ CVD risk } \\
\hline & & OR & $95 \% \mathrm{Cl}$ & & & OR & $95 \% \mathrm{Cl}$ \\
\hline \multirow[t]{4}{*}{$a$-Carotene $(\mu \mathrm{mol} / \mathrm{l})$} & Q1 & 1.00 & - & \multirow[t]{4}{*}{ trans-Lycopene $(\mu \mathrm{mol} / \mathrm{l})$} & Q1 & 1.00 & - \\
\hline & Q2 & 0.64 & $0.53,0.77$ & & Q2 & 0.99 & $0.96,1.02$ \\
\hline & Q3 & 0.65 & $0.54,0.79$ & & Q3 & 1.49 & $0.88,2.50$ \\
\hline & Q4 & 0.47 & $0.38,0.58$ & & Q4 & 1.27 & $0.86,1.88$ \\
\hline \multirow[t]{4}{*}{ trans- $\beta$-Carotene $(\mu \mathrm{mol} / \mathrm{l})$} & Q1 & 1.00 & - & \multirow[t]{4}{*}{ Retinyl palmitate $(\mu \mathrm{mol} / \mathrm{l})$} & Q1 & 1.00 & - \\
\hline & Q2 & 0.64 & $0.52,0.79$ & & Q2 & $1 \cdot 12$ & $0.52,1.35$ \\
\hline & Q3 & 0.64 & $0.56,0.76$ & & Q3 & 1.22 & $0.95,1.63$ \\
\hline & Q4 & 0.66 & $0.43,0.71$ & & Q4 & $1 \cdot 10$ & $0.98,1.42$ \\
\hline \multirow[t]{4}{*}{ cis- $\beta$-Carotene $(\mu \mathrm{mol} / \mathrm{l})$} & Q1 & 1.00 & - & \multirow[t]{4}{*}{ Retinyl stearate $(\mu \mathrm{mol} / \mathrm{l})$} & Q1 & 1.00 & - \\
\hline & Q2 & 0.69 & $0.52,0.79$ & & Q2 & 1.02 & $0.76,1.28$ \\
\hline & Q3 & 0.64 & $0.48,0.75$ & & Q3 & $1 \cdot 14$ & $0.99,1.32$ \\
\hline & Q4 & 0.44 & $0.32,0.55$ & & Q4 & 1.09 & $0.99,1.23$ \\
\hline \multirow[t]{4}{*}{$\beta$-Cryptoxanthin $(\mu \mathrm{mol} / \mathrm{l})$} & Q1 & 1.00 & - & \multirow[t]{4}{*}{ Retinol $(\mu \mathrm{mol} / \mathrm{l})(\operatorname{vitamin} \mathrm{A})$} & Q1 & 1.00 & - \\
\hline & Q2 & 0.85 & $0.73,1.03$ & & Q2 & 0.69 & $0.52,0.79$ \\
\hline & Q3 & 0.98 & $0.77,1.13$ & & Q3 & 0.64 & $0.48,0.75$ \\
\hline & Q4 & 0.81 & $0.67,1.09$ & & Q4 & 0.44 & $0.32,0.55$ \\
\hline \multirow[t]{4}{*}{ Combined lutein/zeaxanthin $(\mu \mathrm{mol} / \mathrm{l})$} & Q1 & 1.00 & - & \multirow[t]{4}{*}{$a$-Tocopherol $(\mu \mathrm{mol} / \mathrm{l})($ vitamin E) } & Q1 & 1.00 & - \\
\hline & Q2 & 0.95 & $0.78,1.12$ & & Q2 & 0.84 & $0.71,1.03$ \\
\hline & Q3 & 0.88 & $0.75,1.29$ & & Q3 & 0.72 & $0.58,0.86$ \\
\hline & Q4 & 0.89 & $0.74,1.25$ & & Q4 & 0.49 & $0.41,0.59$ \\
\hline
\end{tabular}

supplementation (25000 IU/d) was shown to reduce CRP levels in obese women ${ }^{(53)}$. In contrast, Filteau et $a l^{(54)}$ reported increased serum CRP concentrations after supplementation of 200000 IU/d retinyl palmitate for 4 months in children with marginal vitamin A deficiency.

A study involving non-smoking participants from the third NHANES ( $n 4557$ aged $25-55$ years) reported that $\beta$-cryptoxanthin and FG were inversely associated ${ }^{(55)} \cdot \beta$-Cryptoxanthin is plentiful in foods that also tend to be high in vitamin $\mathrm{C}$, which itself has been shown to be inversely related to FG levels ${ }^{(56)}$. Furthermore, Iribarren et $a l .{ }^{(57)}$ found that sialic acid, which is elevated during the acute phase response, and white blood cell count, but not FG levels, were inversely associated with serum $\beta$-carotene levels. In multiple regression analysis, including a number of correlates of $\beta$-carotene levels, sialic acid remained inversely associated with $\beta$-carotene levels ${ }^{(57)}$.

Due to the sampling strategy of NHANES, our findings can be generalised to the US population. However, a principal limitation of this analysis is its cross-sectional nature which cannot allow reliable definition of the direction of the effect of the observed associations. Although we accounted for several lifestyle factors, the possibility of the effect of unmeasured confounders remains. We included on CRP and FG, which may not capture all major inflammatory pathways. Although the sample size was acceptable, it included only a sub-sample of participants who took part in the targeted NHANES surveys. A major strength of this study is the use of objectively measured biomarkers in the analysis rather than relying on self-reported dietary intake. 
In conclusion, the present study supports a possible beneficial effect of antioxidant vitamins on subclinical inflammation, mediated at least in part by the overall adiposity. To what extent, the observed associations may translate into a protective effect of antioxidant vitamins against pathological conditions involving subclinical inflammation, needs to be further investigated.

\section{Acknowledgements}

This research received no specific grant from any funding agency, commercial or not-for-profit sectors.

M. M. contributed to the study concept and design, data analysis and interpretation, and drafting of the manuscript and contributed to the critical revision of the manuscript. A. P. K. contributed to the data analysis and interpretation, and drafting of the manuscript, contributed to the study concept and design. M. B., N. K. and D. P. M. contributed to the critical revision of the manuscript, contributed to the study concept and design. All the authors approved the final version of the paper.

N. K. has given talks, attended conferences and participated in trials sponsored by Amgen, Angelini, AstraZeneca, Boehringer Ingelheim, MSD, Novartis, Novo Nordisk, Sanofi and WinMedica. D. P. M. has given talks and attended conferences sponsored by MSD, AstraZeneca and Libytec. The other authors have no conflict of interest to declare.

\section{References}

1. Szmitko PE, Wang CH, Weisel RD, et al. (2003) New markers of inflammation and endothelial cell activation: part I. Circulation 108, 1917-1923.

2. Pepys MB \& Hirschfield GM (2003) C-reactive protein: a critical update. J Clin Invest 111, 1805-1812.

3. Mazidi M, Heidari-Bakavoli A, Khayyatzadeh SS, et al. (2016) Serum hs-CRP varies with dietary cholesterol, but not dietary fatty acid intake in individuals free of any history of cardiovascular disease. Eur J Clin Nutr 70, 1454-1457.

4. Mazidi M, Karimi E, Rezaie P, et al. (2017) Treatment with GLP1 receptor agonists reduce serum CRP concentrations in patients with type 2 diabetes mellitus: a systematic review and meta-analysis of randomized controlled trials. I Diabetes Complications 31, 1237-1242.

5. Mineo C, Gormley AK, Yuhanna IS, et al. (2005) FcgammaRIIB mediates C-reactive protein inhibition of endothelial $\mathrm{NO}$ synthase. Circ Res 97, 1124-1131.

6. Stefanadi E, Tousoulis D, Papageorgiou N, et al. (2010) Inflammatory biomarkers predicting events in atherosclerosis. Curr Med Chem 17, 1690-1707.

7. Danesh J, Lewington S, Thompson SG, et al. (2005) Plasma fibrinogen level and the risk of major cardiovascular diseases and nonvascular mortality: an individual participant metaanalysis. JAMA 294, 1799-1809.

8. Espinola-Klein C, Rupprecht HJ, Bickel C, et al. (2007) Inflammation, atherosclerotic burden and cardiovascular prognosis. Atherosclerosis 195, e126-e134.

9. Becker RC, Cannon CP, Bovill EG, et al. (1996) Prognostic value of plasma fibrinogen concentration in patients with unstable angina and non-Q-wave myocardial infarction (TIMI IIIB trial). Am J Cardiol 78, 142-147.

10. Hermsdorff HH, Zulet MA, Puchau B, et al. (2010) Fruit and vegetable consumption and proinflammatory gene expression from peripheral blood mononuclear cells in young adults: a translational study. Nutr Metab (Lond) 7, 42.

11. Pellegrini N, Salvatore S, Valtuena S, et al. (2007) Development and validation of a food frequency questionnaire for the assessment of dietary total antioxidant capacity. J Nutr $\mathbf{1 3 7}$, 93-98.

12. Kaur G, Rao LV, Agrawal A, et al. (2007) Effect of wine phenolics on cytokine-induced C-reactive protein expression. $J$ Thromb Haemost 5, 1309-1317.

13. El-Mowafy AM, El-Mesery ME, Salem HA, et al. (2010) Prominent chemopreventive and chemoenhancing effects for resveratrol: unraveling molecular targets and the role of C-reactive protein. Chemotherapy 56, 60-65.

14. Suzuki K, Inoue T, Hashimoto S, et al. (2010) Association of serum carotenoids with high molecular weight adiponectin and inflammation markers among Japanese subjects. Clin Chim Acta 411, 1330-1334.

15. Vivekananthan DP, Penn MS, Sapp SK, et al. (2003) Use of antioxidant vitamins for the prevention of cardiovascular disease: meta-analysis of randomised trials. Lancet 361, 2017-2023.

16. Kushi LH, Folsom AR, Prineas RJ, et al. (1996) Dietary antioxidant vitamins and death from coronary heart disease in postmenopausal women. $N$ Engl J Med 334, 1156-1162.

17. Katsiki N \& Manes C (2009) Is there a role for supplemented antioxidants in the prevention of atherosclerosis? Clin Nutr 28, 3-9.

18. Mazidi M, Katsiki N, Mikhailidis DP, et al. (2018) The link between insulin resistance parameters and serum uric acid is mediated by adiposity. Atherosclerosis 270, 180-186.

19. Mazidi M, Shivappa N, Wirth MD, et al. (2018) Dietary inflammatory index and cardiometabolic risk in US adults. Atherosclerosis 276, 23-27.

20. Centers for Disease Control and Prevention (2015) National Center for Health Statistics, National Health and Nutrition Survey. Survey E. http://www.cdc.gov/nchs/nhanes/ (accessed October 2015)

21. Mazidi M, Michos ED \& Banach M (2017) The association of telomere length and serum 25-hydroxyvitamin D levels in US adults: the National Health and Nutrition Examination Survey. Arch Med Sci 13, 61-65.

22. Mazidi M, Penson P \& Banach M (2017) Association between telomere length and complete blood count in US adults. Arch Med Sci 13, 601-605.

23. Friedewald WT, Levy RI \& Fredrickson DS (1972) Estimation of the concentration of low-density lipoprotein cholesterol in plasma, without use of the preparative ultracentrifuge. Clin Chem 18, 499-502.

24. Pearson TA, Mensah GA, Alexander RW, et al. (2003) Markers of inflammation and cardiovascular disease: application to clinical and public health practice: a statement for healthcare professionals from the Centers for Disease Control and Prevention and the American Heart Association. Circulation 107, 499-511.

25. Mazidi M, Shivappa N, Wirth MD, et al. (2017) The association between dietary inflammatory properties and bone mineral density and risk of fracture in US adults. Eur J Clin Nutr $\mathbf{7 1}$, $1273-1277$.

26. Mazidi M, Kengne AP, Mikhailidis DP, et al. (2017) Dietary food patterns and glucose/insulin homeostasis: a crosssectional study involving 24,182 adult Americans. Lipids Health Dis 16, 192.

27. Slinker BK \& Glantz SA (1985) Multiple regression for physiological data analysis: the problem of multicollinearity. Am J Physiol 249, R1-R12.

28. Preacher KJ \& Hayes AF (2008) Asymptotic and resampling strategies for assessing and comparing indirect 
effects in multiple mediator models. Behav Res Methods $\mathbf{4 0}$, 879-891.

29. Serafini M \& Peluso I (2016) Functional foods for health: the interrelated antioxidant and anti-inflammatory role of fruits, vegetables, herbs, spices and cocoa in humans. Curr Pharm Des 22, 6701-6715.

30. Dagenais GR, Marchioli R, Yusuf S, et al. (2000) Beta-carotene, vitamin $\mathrm{C}$, and vitamin $\mathrm{E}$ and cardiovascular diseases. Curr Cardiol Rep 2, 293-299.

31. Lee IM, Cook NR, Gaziano JM, et al. (2005) Vitamin E in the primary prevention of cardiovascular disease and cancer: the Women's Health Study: a randomized controlled trial. JAMA 294, 56-65.

32. Sleight $\mathrm{P}$ (2000) The HOPE Study (Heart Outcomes Prevention Evaluation). J Renin Angiotensin Aldosterone Syst 1, 18-20.

33. Sesso HD, Christen WG, Bubes V, et al. (2012) Multivitamins in the prevention of cardiovascular disease in men: the Physicians' Health Study II randomized controlled trial. JAMA 308, 1751-1760.

34. Ye Y, Li J \& Yuan Z (2013) Effect of antioxidant vitamin supplementation on cardiovascular outcomes: a meta-analysis of randomized controlled trials. PLOS ONE 8, e56803.

35. Carr BR, Khan N, Adams-Huet B, et al. (2006) Effect of vitamin E supplementation with and without hormone therapy on circulatory inflammatory markers in postmenopausal women. Fertil Steril 85, 667-673.

36. Devaraj S, Li D \& Jialal I (1996) The effects of alpha tocopherol supplementation on monocyte function. Decreased lipid oxidation, interleukin 1 beta secretion, and monocyte adhesion to endothelium. J Clin Invest $\mathbf{9 8}, 756-763$.

37. van Tits LJ, Demacker PN, de Graaf J, et al. (2000) Alpha-tocopherol supplementation decreases production of superoxide and cytokines by leukocytes ex vivo in both normolipidemic and hypertriglyceridemic individuals. Am J Clin Nutr 71, 458-464.

38. Block G, Jensen C, Dietrich M, et al. (2004) Plasma C-reactive protein concentrations in active and passive smokers: influence of antioxidant supplementation. J Am Coll Nutr 23, 141-147.

39. Bruunsgaard H, Poulsen HE, Pedersen BK, et al. (2003) Longterm combined supplementations with alpha-tocopherol and vitamin $C$ have no detectable anti-inflammatory effects in healthy men. J Nutr 133, 1170-1173.

40. Hartel C, Strunk T, Bucsky P, et al. (2004) Effects of vitamin C on intracytoplasmic cytokine production in human whole blood monocytes and lymphocytes. Cytokine 27, 101-106.

41. Baeuerle PA \& Henkel T (1994) Function and activation of NFkappa B in the immune system. Ann Rev Immunol 12, 141-179.

42. Bowie AG \& O'Neill LA (2000) Vitamin C inhibits NF-kappa B activation by TNF via the activation of $\mathrm{p} 38$ mitogen-activated protein kinase. J Immunol 165, 7180-7188.

43. Carcamo JM, Pedraza A, Borquez-Ojeda O, et al. (2002) Vitamin C suppresses TNF alpha-induced NF kappa B activation by inhibiting I kappa B alpha phosphorylation. Biochemistry 41, 12995-13002.

44. Perez-Cruz I, Carcamo JM \& Golde DW (2003) Vitamin C inhibits FAS-induced apoptosis in monocytes and U937 cells. Blood 102, 336-343.

45. Ribeiro Nogueira C, Ramalho A, Lameu E, et al. (2009) Serum concentrations of vitamin A and oxidative stress in critically ill patients with sepsis. Nutr Hosp 24, 312-317.

46. Britton G (1995) Structure and properties of carotenoids in relation to function. FASEB J 9, 1551-1558.

47. Erlinger TP, Guallar E, Miller ER 3rd, et al. (2001) Relationship between systemic markers of inflammation and serum betacarotene levels. Arch Intern Med 161, 1903-1908.

48. Hu P, Reuben DB, Crimmins EM, et al. (2004) The effects of serum beta-carotene concentration and burden of inflammation on all-cause mortality risk in high-functioning older persons: MacArthur studies of successful aging. J Gerontol A Biol Sci Med Sci 59, 849-854.

49. Gawron-Skarbek A, Guligowska A, Prymont-Przyminska A, et al. (2017) Dietary vitamin C, E and beta-carotene intake does not significantly affect plasma or salivary antioxidant indices and salivary C-reactive protein in older subjects. Nutrients 9, E729.

50. Il'yasova D, Ivanova A, Morrow JD, et al. (2008) Correlation between two markers of inflammation, serum C-reactive protein and interleukin 6 , and indices of oxidative stress in patients with high risk of cardiovascular disease. Biomarkers 13, 41-51.

51. McDonald SL, Savy M, Fulford AJ, et al. (2014) A double blind randomized controlled trial in neonates to determine the effect of vitamin A supplementation on immune responses: the Gambia protocol. BMC Pediatr 14, 92.

52. Kim CH (2011) Retinoic acid, immunity, and inflammation. Vitam Horm 86, 83-101.

53. Farhangi MA, Keshavarz SA, Eshraghian M, et al. (2013) Vitamin A supplementation, serum lipids, liver enzymes and C-reactive protein concentrations in obese women of reproductive age. Ann Clin Biochem 50, 25-30.

54. Filteau SM, Morris SS, Raynes JG, et al. (1995) Vitamin A supplementation, morbidity, and serum acute-phase proteins in young Ghanaian children. Am J Clin Nutr 62, 434-438.

55. Kritchevsky SB, Bush AJ, Pahor M, et al. (2000) Serum carotenoids and markers of inflammation in nonsmokers. $A m \mathrm{~J}$ Epidemiol 152, 1065-1071.

56. Khaw KT \& Woodhouse P (1995) Interrelation of vitamin C, infection, haemostatic factors, and cardiovascular disease. BMJ 310, 1559-1563.

57. Iribarren C, Folsom A, Jacobs DR Jr, et al. (1997) Patterns of covariation of serum $\beta$-carotene and $\alpha$-tocopherol in middleaged adults: the Atherosclerosis Risk in Communities (ARIC) Study. Nutr Metab Cardiovasc Dis 7, 445-458. 